\title{
Interrelationship between micronutrients and cardiovascular structure and function in type 2 diabetes
}

\author{
Grace W. M. Walters ${ }^{1,2}\left(0\right.$, Emma Redman ${ }^{2,3}$, Gaurav S. Gulsin ${ }^{1,2 *}$, Joseph Henson ${ }^{2,3}$, \\ Stavroula Argyridou ${ }^{3}$, Thomas Yates ${ }^{2,3}$, Melanie J. Davies ${ }^{2,3}$, Kelly Parke ${ }^{4}$, Gerry P. McCann ${ }^{1,2}$ and \\ Emer M. Brady ${ }^{1,2}$ \\ ${ }^{1}$ Department of Cardiovascular Sciences, University of Leicester, Leicester, UK \\ ${ }^{2}$ National Institute for Health Research (NIHR) Leicester Biomedical Research Centre, Leicester, UK \\ ${ }^{3}$ Diabetes Research Centre, University of Leicester, Leicester, UK \\ ${ }^{4}$ University Hospitals of Leicester NHS Trust, Leicester, UK
}

(Received 1 September 2021 - Accepted 8 September 2021)

Journal of Nutritional Science (2021), vol. 10, e88, page 1 of 9

doi:10.1017/jns.2021.82

Abstract

Micronutrients are important for normal cardiovascular function. They may play a role in the increased risk of cardiovascular disease observed in people with type 2 diabetes (T2D) and T2D-related heart failure. The aims of this study were to (1) examine micronutrient status in people with T2D $v$. healthy controls; (2) assess any changes following a nutritionally complete meal replacement plan (MRP) compared with routine care; (3) determine if any changes were associated with changes in cardiovascular structure/function. This was a secondary analysis of data from a prospective, randomised, open-label, blinded end-point trial of people with T2D, with a nested case-control [NCT02590822]. Anthropometrics, cardiac resonance imaging and fasting blood samples (to quantify vitamins $\mathrm{B}_{1}, \mathrm{~B}_{6}, \mathrm{~B}_{12}, \mathrm{D}$ and $\mathrm{C}$; and iron and ferritin) were collected at baseline and 12 weeks following the MRP or routine care. Comparative data in healthy controls were collected at baseline. A total of eighty-three people with T2D and thirty-six healthy controls were compared at baseline; all had micronutrient status within reference ranges. Vitamin $\mathrm{B}_{1}$ was higher $(148 \cdot 9 v .131 \cdot 7 ; P 0 \cdot 01)$ and $\mathrm{B}_{6}$ lower $(37 \cdot 3 v .52 \cdot 9 ; P 0 \cdot 01)$ in $\mathrm{T} 2 \mathrm{D} v$. controls. All thirty participants randomised to routine care and twenty-four to the MRP completed the study. There was an increase in vitamins $\mathrm{B}_{1}, \mathrm{~B}_{6}, \mathrm{D}$ and $\mathrm{C}$ following the MRP, which were not associated with changes in cardiovascular structure/function. In conclusion, changes in micronutrient status following the MRP were not independently associated with improvements in cardiovascular structure/function in people with T2D.

Key words: Cardiovascular function: CMR: Low calorie: Low-energy meal replacement plan: Micronutrients: Type 2 diabetes

\section{Introduction}

Type-2 diabetes (T2D) is a global epidemic, with the current estimated prevalence in the UK being 4.7 million (of which $90 \%$ is T2D $)^{(1)}$. T2D is a progressive and chronic disease of which cardiovascular complications are leading causes of morbidity and mortality. It is characterised by dysglycaemia, resultant from a combination of insulin resistance and impaired insulin secretion, and confers a two- to four fold increased risk of heart failure $(\mathrm{HF})^{(2)}$ even after adjustment for the prevalence of other traditional risk factors, such as sex, age, hypertension, coronary artery disease, and dyslipidaemia, which are inherent among people with $\mathrm{T} 2 \mathrm{D}^{(3)}$. The structural and functional cardiovascular abnormalities typically described in T2D include concentric left ventricular (LV) remodelling and diastolic dysfunction, which are evident in asymptomatic individuals and recognised as indicators of

Abbreviations: BMI: body mass index; CMR: cardiac magnetic resonance imaging; CVD: cardiovascular disease; EF: ejection fraction; HF: heart failure; LV: left ventricular; MRP: meal replacement plan; PLP: pyridoxal 5-phosphate; RCT: randomised control trial; T2D: type 2 diabetes

* Corresponding author: Gaurav Gulsin, email gg149@leicester.ac.uk 
early or impending $\mathrm{HF}^{(4)}$ (American Heart Association (AHA) stage $\left.\mathrm{B}^{(5,6)}\right)$.

Multiple micronutrients are essential for normal cardiovascular function, a number of which are involved in pathways that modulate inflammation and oxidative damage and thus are thought to play a role in reducing cardiovascular risk ${ }^{(7)}$. Dietary micronutrient deficiency is common in adults with $\mathrm{HF}^{(8)}$. Micronutrients are necessary cofactors for normal cardiac metabolism, and deficiencies have been implicated in the development and progression of $\mathrm{HF}^{(9)}$.

Deficiencies in a variety of micronutrients have also been associated with the development and progression of T2D. Vitamin $\mathrm{B}_{1}$ is implicated in insulin synthesis and secretion impairment ${ }^{(10)}$ and vitamin $B_{6}$ in insulin resistance ${ }^{(11)}$, and $\mathrm{B}_{12}$ deficiency reportedly occurs as a consequence of prolonged metformin use ${ }^{(12)}$. Vitamin D has important effects on insulin action and may impact on a number of pathways with putative importance in the development of $\mathrm{T} 2 \mathrm{D}^{(13)}$. As does iron, which plays a direct and causal role in T2D pathogenesis, mediated by both $\beta$-cell failure and insulin resistance $^{(14)}$.

There is a clear link between T2D and HF and while the prevalence of T2D among the general population in UK is approximately $7 \cdot 1 \%{ }^{(15)}$, among patients with HF the prevalence of diabetes is estimated to be between 35 and 45 $\%^{(16)}$. Given that dietary micronutrient deficiency is common in adults with both $\mathrm{HF}^{(17)}$ and obesity ${ }^{(18)}$, along with the purported role of a number of micronutrients in the pathogenesis and progression of $\mathrm{T} 2 \mathrm{D}^{(19)}$, and indeed the development of HF; it could be suggested that the association between T2D and HF may be, in part, a result of nutrient deficiencies common to $\mathrm{HF}$ and $\mathrm{T} 2 \mathrm{D}$.

Modifiable risk factors, including nutritional alteration, are ever more important with the increasing prevalence of chronic and progressive conditions such as T2D. The identification and correction of micronutrient deficiencies may offer potential therapeutic roles in the prevention and treatment of T2D-related cardiovascular disease (CVD) including HF.

Therefore, the aim of the present paper was to examine circulating levels of vitamin $B_{1}$, vitamin $B_{6}$, vitamin $B_{12}$, vitamin $\mathrm{C}$, vitamin $\mathrm{D}$, iron and ferritin in a cohort of people with $\mathrm{T} 2 \mathrm{D}$ with the evidence of subclinical cardiovascular dysfunction. While other micronutrients such as zinc ${ }^{(20)}$ and vitamin $\mathrm{E}^{(21)}$ have been associated with heart disease and CVD prevention, these specific micronutrients are not considered in the present study as they were not analysed from the fasting blood samples.

The analysis was a three-part analysis investigating the following: (1) micronutrient status in people with T2D $v$. healthy controls; (2) any changes in micronutrient status following a nutritionally complete meal replacement plan (MRP) compared with routine care and (3) if any changes were associated with changes in cardiovascular structure and function.

\section{Methods}

The rationale, design and main study findings of the 'Diabetes Interventional Assessment of Slimming or Training to Lessen
Inconspicuous Cardiovascular dysfunction' (DIASTOLIC) trial have been previously reported ${ }^{(22,23)}$. In brief, DIASTOLIC was a prospective, randomised, open-label, blinded end-point trial assessing the effects of a low-calorie MRP $v$. exercise training $v$. routine care in working-age adults with T2D and obesity. This study was conducted according to the guidelines laid down in the Declaration of Helsinki, and all procedures involving human subjects/patients were approved by the National Research Ethics Service (15/WM/0222). All participants provided written informed consent.

\section{Participants}

The participants included in the DIASTOLIC trial were aged 18-65 years with established T2D (duration $>3$ months) diagnosed before age 60 years with a body mass index (BMI) of $>30 \mathrm{~kg} / \mathrm{m}^{2}$ (or $>27 \mathrm{~kg} / \mathrm{m}^{2}$ if South Asian or black ethnicity). Participants were ineligible to participate if T2D duration was $>12$ years, they were on the current treatment of more than three glucose-lowering medications or insulin, had history, signs or symptoms of CVD (including coronary artery disease, stroke, transient ischaemic attack, peripheral artery disease or $\mathrm{HF}$ ) and had weight loss of $>5 \mathrm{~kg}$ in the preceding 6 months or an inability to exercise or undertake the MRP. Healthy volunteers free of T2D, obesity, hypertension or confirmed CVD were recruited for baseline case-control comparison. A full list of the inclusion and exclusion criteria is provided within Supplementary Material, Document 1 . Those participants who fit the inclusion criteria were randomised to one of the three arms of the intervention group. Age-, sex- and ethnicity-matched healthy volunteers were also recruited for a one time point data collection (equivalent to baseline) for baseline case-control comparisons. See the CONSORT diagram (Fig. 1) for participant numbers enrolled, completion of randomised control trial (RCT) and numbers included in each analysis.

\section{Interventions}

The DIASTOLIC study had three trial arms: routine care, a nutritionally complete MRP and a supervised aerobic exercise programme; details of which can be found in Supplementary Material, Document 2. Participants were randomised (1:1:1) to one of these groups for 12 weeks. For this secondary analysis, we only focused on the routine care and MRP arms because the exercise arm did not alter participants' micronutrient intake as part of that intervention. The dietary arm followed a low-energy, nutritionally complete MRP, providing all the reference nutrient intakes of micronutrients ${ }^{(24)}$, for 12 weeks which comprised an average of $\sim 810 \mathrm{kcal} / \mathrm{d}$ (30 \% proteins, $50 \%$ carbohydrates and $20 \%$ fats) (Cambridge Weight Plan).

\section{Assessments}

Participants with T2D in the trial underwent two main assessment visits: baseline and 12 weeks. Each visit consisted of the following assessments: demographics, medical history, 


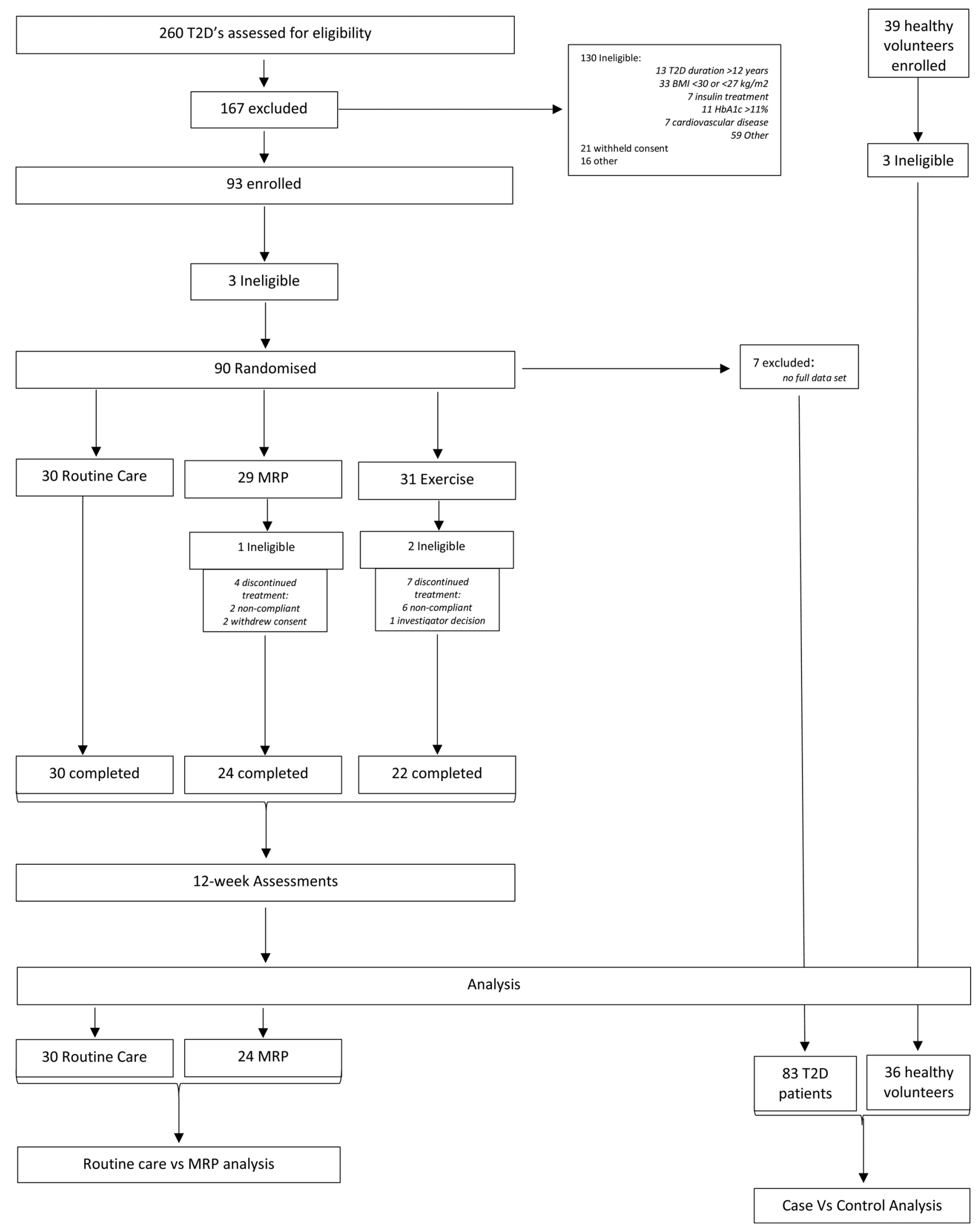

Fig. 1. CONSORT diagram. MRP, meal replacement plan; T2D, type 2 diabetes.

bio-anthropometrics (height, weight, BMI, hip:waist ratio, fasting blood sample and blood pressure), comprehensive cardiovascular magnetic resonance $(\mathrm{CMR})$ imaging and a symptom-limited incremental cardio-pulmonary exercise test. The data collected were identical for healthy volunteers and participants with T2D and obesity. 
Fasting blood sample analysis and micronutrient quantification

A fasting venous blood sample was obtained to assess the biochemical profiles for T2D control (glucose and HbA1c) and micronutrients. All fasting blood samples were analysed in an NHS accredited laboratory, and the details of this biochemical analysis can be found in Supplementary Material, Document 3.

\section{Cardiovascular magnetic resonance}

In addition, participants had comprehensive CMR imaging on a $1.5 \mathrm{~T}$ field strength scanner (MAGNETOM Aera; Siemens, Erlangen, Germany) using a standardised protocol ${ }^{(22)}$. CMR images were analysed offline while blinded to all participant details and the treatment group by experienced operators.

\section{Statistical analysis}

Statistical analyses were performed using a commercially available software package version 26 (IBM SPSS Statistics, Hampshire, UK). The normality of the outcome measures was assessed using histograms, the Shapiro-Wilk test and Q-Q plots. Independent samples $t$ tests or Mann-Whitney tests were employed to determine any differences in micronutrients between cases and controls. Data were split according to the treatment group and paired samples $t$ tests or Wilcoxon test were used to determine if micronutrients were different from baseline to 12 weeks within the MRP and the routine care group. Independent samples $t$ tests or MannWhitney tests were then used to determine if the change from baseline to 12 weeks was different between the MRP group and the routine care group. To determine if micronutrient status was correlated with cardiac structure and function variables, bivariate Spearman's correlation estimates were produced using baseline data for the cohort of participants with obesity and T2D. The cardiovascular structure/function variables of interest were those that had previously been described as being improved following the 12 -week $\mathrm{MRP}^{(23)}$ :

(1) Change in LV mass to volume ratio ( $\mathrm{LV}$ mass:volume, $\mathrm{g} / \mathrm{ml}$ ).

(2) Change in mean aortic distensibility $\left(\mathrm{mmHg}^{-1} \times 10^{-3}\right)$.

The micronutrients that were significantly different from baseline to 12 weeks in the MRP arm only were modelled (using linear regression) against the cardiac structure/function variables. An unadjusted, and baseline-adjusted, univariate model was used to assess if there was any association between changes in micronutrients and changes in cardiovascular structure/function variables. Data are expressed as mean ( \pm standard deviation) for normally distributed data and median (interquartile range) for not normally distributed data. A $P$ value $<0.05$ was considered statistically significant.

\section{Results}

A total of eighty-three people with T2D and obesity and thirtysix healthy volunteers were included in the case $v$. control baseline analysis (Table 1). See the CONSORT diagram (Fig. 1) for participant numbers enrolled, completion of RCT and numbers included in each analysis. Intervention groups were well balanced with no significant differences between people in the MRP or routine care arms at baseline, for age, sex, ethnicity, body weight, BMI or HbA1c concentration, or any of the micronutrients at baseline (Table 2). Of the eighty-three participants in the trial, a total of seventy-six completed the intervention, fifty-four of which were in either the MRP ( $n$ 24) or routine care arm (n 30) with no significant differences in characteristics at baseline (Table 2).

Only participants with a full data set at baseline were included in the analysis; therefore, three participants with T2D and obesity were excluded from the case $v$. control baseline analysis due to missing data.

\section{Case-control comparison}

The healthy volunteer (control) and T2D with obesity group (case) were similar for age, sex and ethnicity distribution; however, the control group had lower overall body weight and BMI, blood pressure, heart rate and HbA1c (Table 1). Micronutrient levels for both groups were within reference ranges (Supplementary Table S1) ${ }^{(25,26)}$.

Vitamin $\mathrm{B}_{1}$ was higher in cases compared with controls $(148 \cdot 87 \pm 30.64$ v. $131 \cdot 71 \pm 25 \cdot 25 \mathrm{nmol} / 1, \quad P \quad 0 \cdot 01)$, while levels of vitamin $\mathrm{B}_{6}$ were lower in cases compared with controls $(37.28 \pm 22.01 \quad v$. $52.94 \pm 23.83 \mathrm{nmol} / 1$, respectively; P 0.001) (Table 1). Vitamin C, vitamin $\mathrm{B}_{12}$, vitamin $\mathrm{D}$, iron and ferritin showed no significant differences between cases and controls (Table 1).

\section{Pre- and post 12-week MRP}

Twelve-week changes in bio-anthropometrics, LV mass:volume and aortic distensibility in the two trial arms are presented in Supplementary Table S1 and Document 4. In brief, following the 12-week MRP, body weight was reduced, and blood pressure, glycaemic status, LV mass:volume ratio and aortic distensibility all improved (all $P<0 \cdot 05$ ). The routine care arm also showed a significant reduction in weight (median [QR] $-1.05(-3.16,-0.01))$; however, this was a significantly smaller reduction than the weight reduction seen in the MRP arm. Blood pressure was also reduced in the routine care arm (mean $-7.1 \mathrm{mmHg}$ systolic, $-1.8 \mathrm{mmHg}$ diastolic). No significant changes in cardiovascular structure and function measures were observed following 12 weeks of routine care.

Four micronutrients increased from baseline to 12 weeks following the MRP (Table 3): vitamin $\mathrm{B}_{1}(+16 \cdot 38[ \pm 24 \cdot 20]$; $P$ 0.009), vitamin $\mathrm{B}_{6}(+11.5$ [27.25]; $P$ 0.016), vitamin $\mathrm{D}$ $(+0.50,[1.0] ; P 0.005)$ and vitamin $\mathrm{C}(+12.59$ [41.78]; $P$ $0 \cdot 009)$. Vitamin $B_{12}$, ferritin and iron showed no significant differences from baseline to 12 weeks post-MRP. Vitamin $\mathrm{B}_{6}$ also increased in the routine care arm $(+6 \cdot 00[ \pm 12 \cdot 00] ; P$ $0 \cdot 014$ ) (the percentage change within groups is illustrated in Fig. 2). 
Table 1. Baseline demographics: cases $v$. controls

\begin{tabular}{|c|c|c|c|c|c|c|c|}
\hline \multirow{3}{*}{$\begin{array}{l}\text { Variable } \\
\text { Demographics }\end{array}$} & \multicolumn{2}{|c|}{ Cases } & \multicolumn{2}{|c|}{ Controls } & \multirow[b]{3}{*}{$P$ value } & & \\
\hline & Mean or median & SD or (IQR) & Mean or median & SD or (IQR) & & & \\
\hline & & & & & & & \\
\hline$N$ & 83 & $\mathrm{~N} / \mathrm{A}$ & 36 & N/A & $\mathrm{N} / \mathrm{A}$ & & \\
\hline Age & $50 \cdot 4$ & $6 \cdot 4$ & $48 \cdot 6$ & $6 \cdot 2$ & 0.150 & & \\
\hline Sex (\% male) & $57 \cdot 8 \%$ & $\mathrm{~N} / \mathrm{A}$ & $52.8 \%$ & N/A & 0.552 & & \\
\hline Minority ethnicity $(N(\%))$ & $33(39 \%)$ & $\mathrm{N} / \mathrm{A}$ & $12(33 \%)$ & N/A & 0.51 & & \\
\hline T2D duration (months) & $62 \cdot 4$ & $38 \cdot 2$ & $N / A$ & N/A & $\mathrm{N} / \mathrm{A}$ & & \\
\hline $\mathrm{HbA}_{1 \mathrm{c}}(\mathrm{mmol} / \mathrm{mol})$ & $7 \cdot 3$ & $1 \cdot 0$ & $5 \cdot 4$ & 0.2 & $<0.001$ & & \\
\hline $\mathrm{HbA}_{1 \mathrm{c}}(\%)$ & $56 \cdot 0$ & $11 \cdot 2$ & $35 \cdot 3$ & $3 \cdot 6$ & $<0.001$ & & \\
\hline Weight (kg) & $102 \cdot 4$ & $16 \cdot 0$ & $70 \cdot 4$ & $10 \cdot 8$ & $<0.001$ & & \\
\hline BMI $\left(\mathrm{kg} / \mathrm{m}^{2}\right)$ & $36 \cdot 4$ & $5 \cdot 4$ & $24 \cdot 5$ & $2 \cdot 4$ & $<0.001$ & & \\
\hline $\mathrm{SBP}(\mathrm{mmHg})$ & 139.9 & $15 \cdot 5$ & $120 \cdot 9$ & $13 \cdot 2$ & $<0.001$ & & \\
\hline $\mathrm{DBP}(\mathrm{mmHg})$ & $87 \cdot 6$ & $8 \cdot 0$ & $76 \cdot 4$ & $7 \cdot 2$ & $<0.001$ & & \\
\hline Heart rate (BPM) & $74 \cdot 2$ & $9 \cdot 8$ & $61 \cdot 7$ & $9 \cdot 8$ & $<0.001$ & & \\
\hline \multicolumn{8}{|l|}{ Cardiovascular variables } \\
\hline LV mass:volume ratio $(\mathrm{g} / \mathrm{ml})$ & $0 \cdot 8$ & $0 \cdot 1$ & 0.71 & $0 \cdot 1$ & $<0.001$ & & \\
\hline Aortic distensibility $\left(\mathrm{mmHg}^{-1} \times 10^{-3}\right)$ & $4 \cdot 2$ & $2 \cdot 1$ & $6 \cdot 6$ & $2 \cdot 0$ & $<0.001$ & & \\
\hline Vitamins & & & & & Mean difference & $(95 \% \mathrm{Cl})$ & $P$ value \\
\hline Vitamin $\mathrm{B}_{1}(\mathrm{nmol} / \mathrm{l})$ & $148 \cdot 9$ & $30 \cdot 6$ & $131 \cdot 0$ & $(25 \cdot 0)$ & $17 \cdot 2$ & $(5 \cdot 4,28 \cdot 9)$ & 0.01 \\
\hline Vitamin $\mathrm{B}_{6}(\mathrm{nmol} / \mathrm{l})$ & $32 \cdot 0$ & $(21 \cdot 3)$ & 52.9 & $23 \cdot 8$ & $15 \cdot 7$ & $(24.9,6.4)$ & 0.01 \\
\hline Iron $(\mu \mathrm{mol} / \mathrm{l})$ & 14.5 & $(7 \cdot 0)$ & $16 \cdot 9$ & 6.9 & 1.9 & $(4 \cdot 2,0.4)$ & $0 \cdot 10$ \\
\hline Vitamin D (nmol/l) & $3 \cdot 0$ & $(2 \cdot 0)$ & $3 \cdot 0$ & $(1 \cdot 0)$ & $0 \cdot 2$ & $(0.6,0.1)$ & 0.23 \\
\hline Vitamin $C(\mu \mathrm{mol} / \mathrm{l})$ & $56 \cdot 7$ & $(39 \cdot 3)$ & $47 \cdot 7$ & $20 \cdot 0$ & $6 \cdot 3$ & $(2 \cdot 9,15 \cdot 6)$ & 0.17 \\
\hline Vitamin $B_{12}(\mathrm{ng} / \mathrm{l})$ & $390 \cdot 1$ & $136 \cdot 4$ & $374 \cdot 5$ & $(151 \cdot 3)$ & $10 \cdot 9$ & $(67 \cdot 5,45 \cdot 7)$ & 0.83 \\
\hline Ferritin M $(\mu \mathrm{g} / \mathrm{l})$ & $119 \cdot 5$ & $(92 \cdot 8)$ & $121 \cdot 0$ & $(88.0)$ & $18 \cdot 4$ & $(50 \cdot 4,87 \cdot 2)$ & 0.82 \\
\hline Ferritin F $(\mu \mathrm{g} / \mathrm{l})$ & $46 \cdot 0$ & $(67 \cdot 3)$ & $47 \cdot 2$ & $31 \cdot 8$ & $11 \cdot 9$ & $(13 \cdot 2,37 \cdot 0)$ & 0.41 \\
\hline
\end{tabular}

Abbreviations: BPM, beats per minute; DBP, diastolic blood pressure; $\mathrm{HbA}_{1 \mathrm{c}}$, glycated haemoglobin; mmHg, millimetre of mercury; SBP, systolic blood pressure; T2D, type 2 diabetes; SD, standard deviation; $\mathrm{Cl}$, confidence interval.

Vitamin D showed a trend towards increasing levels in the MRP arm (P 0.06). However, all other micronutrients showed no differences in the change from baseline to 12 weeks between the two arms.

Table 2. Baseline demographics: intervention groups

\begin{tabular}{|c|c|c|c|c|c|}
\hline \multirow{2}{*}{$\begin{array}{l}\text { Variable } \\
\text { Demographics }\end{array}$} & \multicolumn{2}{|c|}{ Routine care } & \multicolumn{2}{|c|}{ MRP } & \multirow{2}{*}{$\begin{array}{c}P \\
\text { value }\end{array}$} \\
\hline & Mean & SD & Mean & SD & \\
\hline$N$ & 30 & & 24 & & \\
\hline Age & $50 \cdot 7$ & $6 \cdot 4$ & $51 \cdot 1$ & $5 \cdot 7$ & 0.81 \\
\hline Sex (male) & $60 \%$ & & $63 \%$ & & 0.86 \\
\hline $\begin{array}{l}\text { Ethnicity }(N(\%)) \text { (White/ } \\
\text { BAME) }\end{array}$ & $18(60 \%)$ & & $15(63 \%)$ & & 0.86 \\
\hline T2D duration (months) & $60 \cdot 5$ & 31.5 & $58 \cdot 3$ & 39.8 & 0.39 \\
\hline $\mathrm{HbA} 1 \mathrm{c}(\mathrm{mmol} / \mathrm{mol})$ & $7 \cdot 3$ & 0.9 & $7 \cdot 2$ & $1 \cdot 1$ & 0.39 \\
\hline Weight (kg) & $102 \cdot 6$ & $14 \cdot 9$ & $106 \cdot 7$ & $16 \cdot 2$ & 0.35 \\
\hline $\mathrm{BMI}\left(\mathrm{kg} / \mathrm{m}^{2}\right)$ & $36 \cdot 8$ & $4 \cdot 8$ & $37 \cdot 3$ & $5 \cdot 8$ & 0.75 \\
\hline Resting SBP (mmHg) & $137 \cdot 8$ & $12 \cdot 7$ & $145 \cdot 9$ & 15.9 & 0.05 \\
\hline Resting DBP (mmHg) & $85 \cdot 3$ & $7 \cdot 3$ & $91 \cdot 1$ & $7 \cdot 4$ & 0.01 \\
\hline Resting heart rate (BPM) & $76 \cdot 3$ & $7 \cdot 5$ & $73 \cdot 1$ & $8 \cdot 6$ & $0 \cdot 15$ \\
\hline \multicolumn{6}{|l|}{ Cardiac structure/function } \\
\hline $\begin{array}{l}\text { LV mass:volume ratio } \\
(\mathrm{g} / \mathrm{ml})\end{array}$ & 0.8 & 0.1 & 0.8 & 0.1 & 0.54 \\
\hline $\begin{array}{l}\text { Aortic distensibility } \\
\left(\mathrm{mmHg}-1 \times 10^{-3}\right)\end{array}$ & $4 \cdot 2$ & 1.9 & $3 \cdot 7$ & 1.9 & 0.22 \\
\hline
\end{tabular}

Abbreviations: BAME, black and minority ethnic; BMI, body mass index; BPM, beats per minute; DBP, diastolic blood pressure; SBP, systolic blood pressure; T2D, type 2 diabetes; SD, standard deviation; IQR, interquartile range.
There were no significant correlations or linear associations between the change in micronutrients and previously observed changes in cardiovascular structure/function (Supplementary Tables S3 and S4). Due to a lack of a significant relationship between the measures of cardiovascular structure/function with the unadjusted model, the only additional model was that which included baseline adjustment for vitamin level which also demonstrated no significant relationship (data not reported).

\section{Discussion}

To our knowledge, this is the first study to investigate the effects of a nutritionally complete MRP on the plasma micronutrient concentrations of people with obesity and established T2D (but asymptomatic AHA stage $\mathrm{B} \mathrm{HF}^{(6)}$ ) and its subsequent impact on cardiovascular structure and function. The major findings of this secondary analysis were that vitamin $\mathrm{B}_{1}$ and $\mathrm{B}_{6}$ levels are different between people with obesity and T2D compared with matched healthy controls. Secondly, following a 12-week intervention with a low-energy MRP and associated dramatic reductions in weight, blood pressure and glycaemia, there were significant increases in vitamins $B_{1}, B_{6}, D$ and $C$. Despite this, these changes were not associated with the previously observed improvements in cardiovascular structure and function.

A key finding from our investigation was that vitamin $B_{1}$ was higher in people with T2D compared with healthy 
Table 3. Vitamin profile change: routine care and MRP

\begin{tabular}{|c|c|c|c|c|c|c|c|}
\hline \multirow[b]{2}{*}{ Variable } & \multicolumn{2}{|c|}{ Baseline } & \multicolumn{2}{|c|}{12 weeks } & \multicolumn{2}{|c|}{ Change } & \multirow[b]{2}{*}{$P$ value } \\
\hline & Mean or median & SD or (IQR) & Mean or median & SD or (IQR) & Mean or median & SD or (IQR) & \\
\hline \multicolumn{8}{|l|}{ Routine care } \\
\hline Vitamin $\mathrm{B}_{1}(\mathrm{nmol} / \mathrm{l})$ & 152.48 & 31.01 & $160 \cdot 87$ & $27 \cdot 15$ & 8.39 & 22.51 & 0.13 \\
\hline Vitamin $B_{6}(\mathrm{nmol} / \mathrm{l})$ & 28.00 & $(23.00)$ & 38.00 & $(18 \cdot 25)$ & 6.00 & $(12 \cdot 00)$ & 0.01 \\
\hline Iron $(\mu \mathrm{mol} / \mathrm{l})$ & 14.00 & $(7.00)$ & 12.50 & $(7 \cdot 75)$ & -1.00 & $(5 \cdot 00)$ & 0.31 \\
\hline Vitamin D (nmol/l) & 3.00 & $(2 \cdot 00)$ & 3.00 & $(1.00)$ & 0.00 & $(2 \cdot 00)$ & 0.81 \\
\hline Vitamin C $(\mu \mathrm{mol} / \mathrm{l})$ & 38.87 & 23.96 & 41.43 & 22.05 & 4.01 & 16.08 & 0.190 \\
\hline Vitamin $\mathrm{B}_{12}(\mathrm{ng} / \mathrm{l})$ & 345.00 & $(111.50)$ & 330.00 & $(164 \cdot 50)$ & 0.00 & $(93 \cdot 00)$ & 0.461 \\
\hline Ferritin $M(\mu \mathrm{g} / \mathrm{l})$ & 113.50 & $(106 \cdot 00)$ & 99.00 & $(85 \cdot 75)$ & -13.50 & $(57.50)$ & 0.56 \\
\hline Ferritin F $(\mu \mathrm{g} / \mathrm{l})$ & 45.00 & $(45 \cdot 00)$ & 51.00 & (64.50) & 2.00 & $(15 \cdot 00)$ & 0.72 \\
\hline \multicolumn{8}{|l|}{ MRP } \\
\hline Iron $(\mu \mathrm{mol} / \mathrm{l})$ & $14 \cdot 31$ & 6.02 & $15 \cdot 31$ & 5.48 & 1.00 & 3.39 & 0.332 \\
\hline Vitamin D (nmol/l) & 3.00 & $(2 \cdot 00)$ & 3.00 & $(1.00)$ & 0.50 & $(1 \cdot 0)$ & 0.005 \\
\hline Vitamin $C(\mu \mathrm{mol} / \mathrm{l})$ & 35.96 & $(42 \cdot 71)$ & $55 \cdot 12$ & $(31.59)$ & 12.59 & $(41.78)$ & 0.009 \\
\hline Vitamin $B_{12}(\mathrm{ng} / \mathrm{l})$ & 402.00 & $(200 \cdot 00)$ & 430.00 & $(205.00)$ & 43.00 & $(141 \cdot 75)$ & 0.10 \\
\hline Ferritin $M(\mu \mathrm{g} / \mathrm{l})$ & 97.00 & $(162 \cdot 00)$ & 106.00 & $(172 \cdot 00)$ & 11.00 & $(87.00)$ & 0.31 \\
\hline Ferritin F $(\mu \mathrm{g} / \mathrm{l})$ & 58.44 & 46.18 & $65 \cdot 00$ & 49.54 & 6.56 & $27 \cdot 35$ & 0.49 \\
\hline
\end{tabular}

Abbreviations: BAME, black and minority ethnic; BMI, body mass index; BPM, beats per minute; DBP, diastolic blood pressure; SBP, systolic blood pressure; T2D, type 2 diabetes; SD, standard deviation, IQR, interquartile range.

Data split according to the treatment group. Paired samples $t$ tests or Wilcoxon test were used to determine if micronutrients were different from baseline to 12 weeks within the MRP and the routine care group.

controls. This was unexpected as previously vitamin $\mathrm{B}_{1}$ deficiency has been reported among people with T2D and is implicated in the development of various complications of advancing diabetes ${ }^{(27)}$. A cross-sectional comparative study of normal controls showed that thiamine (vitamin $\mathrm{B}_{1}$ ) was lower in people with T2 $\mathrm{D}^{(28)}$, and several studies have demonstrated thiamine deficiency in individuals with both type 1 and type 2 diabetes $^{(10)}$. Insulin deficiency is associated with a reduction in the rate of thiamine transport across the intestine and thiamine deficiency leads to a marked impairment in insulin synthesis and secretion; thus, insulin deficiency may exacerbate thiamine deficiency and vice versa ${ }^{(10)}$. B vitamins have been postulated to play a role in CVD prevention and cardiovascular health. Thiamine deficiency is a recognised contributor of new-onset HF of initially uncertain aetiology ${ }^{(29)}$ and a meta-analysis of randomised, double-blind, placebo-controlled

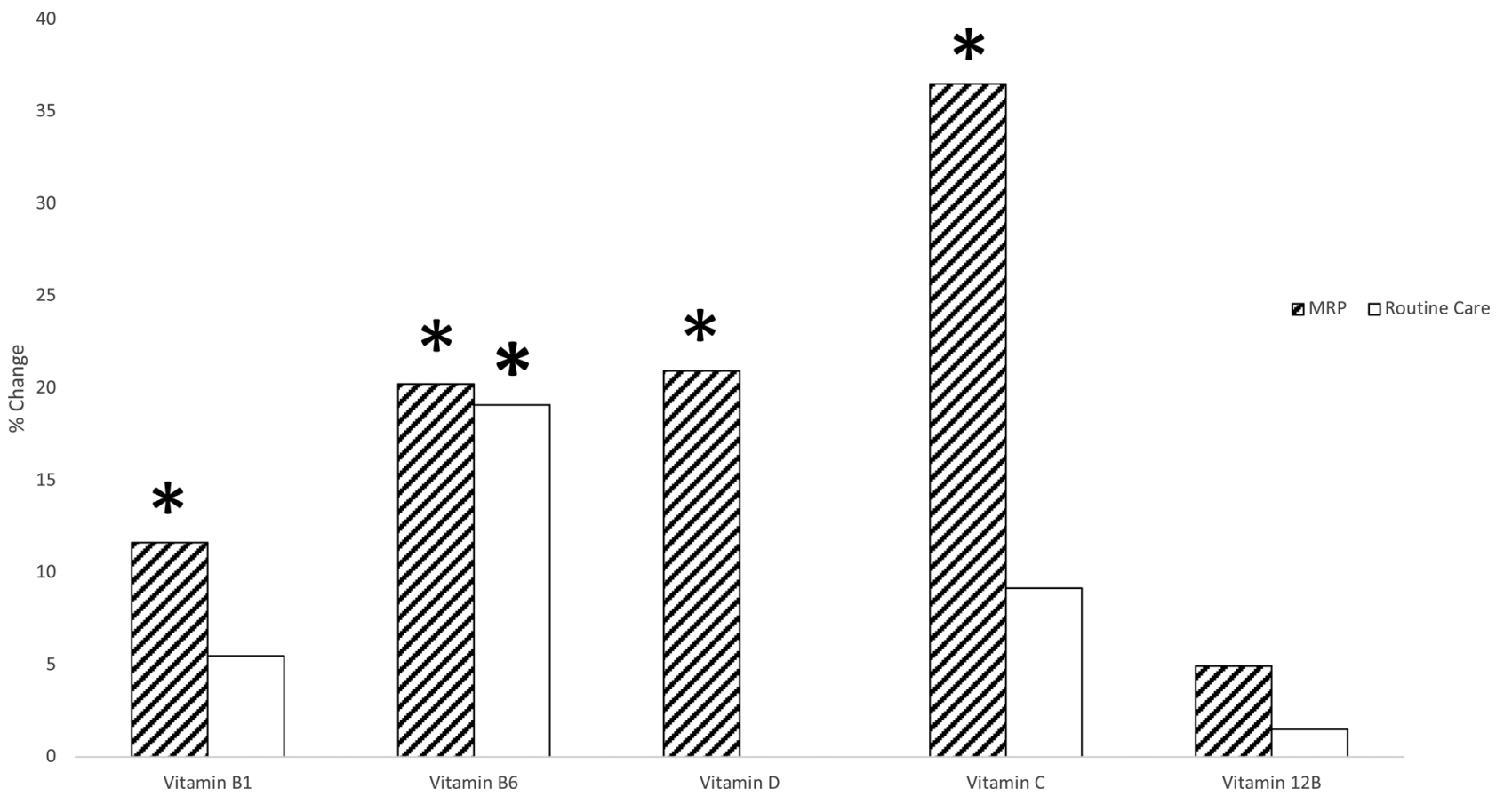

Fig. 2. Comparison of between groups (MRP and routine care) change in micronutrients from baseline to 12 weeks. Data split according to the treatment group. Paired samples $t$ tests or Wilcoxon test were used to determine if micronutrients were different from baseline to 12 weeks within the MRP and the routine care group. Independent samples $t$ tests or Mann-Whitney tests were then used to determine if the change from baseline to 12 weeks was different between the MRP group and the routine care group. *Represents a significant change from baseline to 12 weeks. MRP, meal replacement plan. 
trials reported that thiamine supplementation in patients with systolic HF increased ejection fraction compared with placebo ${ }^{(30)}$. This discrepancy in levels of vitamin $B_{1}$ between our results and the current literature may be a result of the relatively small sample sizes and the relatively young age and short T2D duration within the present cohort. The participants in this study had an average T2D duration of just 62.4 months; this relatively short T2D duration is the most likely result of the participants being younger than in previous studies (the DIASTOLIC study only recruited working-age adults). The higher vitamin $\mathrm{B}_{1}$ level among the participants with T2D and obesity is also likely to have limited clinical relevance because vitamin levels for both groups were within the normal range ${ }^{(25,26)}$. It is, however, worth noting that while vitamin $B_{1}$ was within reference ranges, the T2D participants in the MRP arm of the intervention did see a significant increase in vitamin $B_{1}$. However, the change was not significantly different from the change seen in the routine care arm, therefore adding to the narrative that this increase is likely clinically insignificant.

We also observed lower levels of vitamin $\mathrm{B}_{6}$ in people with $\mathrm{T} 2 \mathrm{D}$ and obesity, which is in line with the previous literature. The deficiency of vitamin $\mathrm{B}_{6}$ has been implicated in $\mathrm{T} 2 \mathrm{D}^{(31)}$ and reduced vitamin $\mathrm{B}_{6}$ availability is implicated in insulin resistance $^{(32)}$. It has been observed that people newly diagnosed with T2D have lower concentrations of the catalytically active part of vitamin $\mathrm{B}_{6}$, pyridoxal $5^{\prime}$-phosphate (PLP) compared with people without T2D ${ }^{(33)}$. PLP is involved in the pathway that converts tryptophan into nicotinic acid ${ }^{(11)}$ and metabolites produced when this pathway does not work properly, partly as a consequence of low levels of PLP, can interfere with insulin activity ${ }^{(11)}$, causing insulin resistance, a hallmark of T2D. PLP deficiency may also impact on insulin resistance by controlling the expression of genes involved in adipogenesis $^{(11)}$, or may cause insulin resistance through an increase of homocysteine due to the impairment of enzymes ${ }^{(11)}$. There is also evidence that vitamin $\mathrm{B}_{6}$ deficiency may negatively affect the progression of $\mathrm{T} 2 \mathrm{D}$ once the disease is present ${ }^{(27)}$, via its contribution to insulin resistance. Insulin resistance and prolonged elevated circulating glucose levels may contribute to the pathogenesis of diabetic cardiomyopathy ${ }^{(4)}$. Insulin resistance and worsening glycaemia are associated with increased LV mass to LV end-diastolic volume ratio (a marker of remodelling) indicating a potential indirect role of $\mathrm{B}_{6}$ deficiency in concentric $\mathrm{LV}$ remodelling ${ }^{(34)}$. The findings of the present study are, however, in conflict with this as an association between the increase in vitamin $\mathrm{B}_{6}$ and the improvement in LV mass:volume ratio was not identified. This lack of association may be a result of the fact that vitamin $\mathrm{B}_{6}$ was within healthy reference ranges at baseline and 12 weeks; therefore, while there was a significant increase in vitamin $B_{6}$ following the 12-week MRP, the concentration of vitamin $\mathrm{B}_{6}$ was always within a 'healthy' range. An association between improvements in vitamin $\mathrm{B}_{6}$ and $\mathrm{LV}$ mass:volume ratio may be present if the concentration of vitamin $B_{6}$ increased from an 'unhealthy' to a 'healthy' range; thus, this lack of association may be associated with the fact that participants did not present with sub-optimal nutritional status.
A significant increase in four micronutrients (vitamins $B_{1}$, $\mathrm{B}_{6}, \mathrm{D}$ and $\mathrm{C}$ ) in the MRP arm was observed. This is the first time to our knowledge that these changes have been observed following a nutritionally complete MRP in people with T2D. This increase was not unexpected as this was a nutritionally complete MRP, providing all the reference nutrient intakes of micronutrients ${ }^{(24)}$. As the observed increase in micronutrients was maintained within reference ranges, it was unlikely to have any clinically significant impact.

The improvement in vitamin D may be of particular importance when considering the role that vitamin $\mathrm{D}$ deficiency is postulated to play in the development and/or progression of chronic $\mathrm{HF}^{(35,36)}$. Vitamin $\mathrm{D}$ deficiency has been shown to be associated with increased LV dilation, assessed by transthoracic echocardiography, in an elderly chronic HF population $^{(37)}$. An LV mass:volume ratio has been shown to be higher (suggestive of concentric LV remodelling) in those with $\mathrm{T} 2 \mathrm{D}^{(38)}$. However, there was no difference in levels of vitamin $\mathrm{D}$ between cases and controls in the present study, despite vitamin $\mathrm{D}$ deficiency being common among this population group ${ }^{(39)}$. This may again be attributed to the health status of the present cohort and may be different for people with a longer duration of, and more poorly controlled, T2D, or those who have additional comorbidities.

Our final finding is that changes in micronutrients were not independently associated with the observed improvements in cardiovascular structure and function, therefore suggesting that there is likely another mechanism(s) responsible for these favourable alterations. Contrary to this, a lack of association could be resultant of the relatively good health status of this particular cohort of participants who were asymptomatic of CVD had a relatively short duration of T2D (62.4 months) which was relatively well controlled (mean HbA1c 7.27\%), and their circulating micronutrients were within reference ranges. Therefore, there may be a limit to the expected level of improvement. Furthermore, the original trial was not powered to detect significant differences in micronutrients between the groups following the interventions; therefore, the observed results could be resultant from type II error ${ }^{(40)}$. A further limitation of this investigation was that while the participants' micronutrient supplementation at baseline was captured in the case report forms as part of the medical history, it was not collected robustly. The information provided did not allow us to standardise or confirm what combination of nutrients, quantity, frequency or quality of supplement. Therefore, this was not included as a confounder in the univariate model that was used to assess if there was any association between changes in micronutrients and changes in cardiovascular structure/function variables. This, therefore, warrants further investigation in a larger, sufficiently powered cohort that includes older symptomatic adults, with longer duration or less well-controlled T2D. There is also potential value in identifying people with T2D and obesity with poor nutritional status and investigating the effects of such an intervention.

An alternative mechanism responsible for the improvements in LV mass:volume ratio and aortic distensibility that we observed in the MRP group may be centred on the reversal of the haemodynamic alterations that occur with loss of excess 
body weight. Weight loss is associated with reductions in many of the haemodynamic alterations that develop with obesity ${ }^{(41)}$. Obesity exerts numerous effects on the cardiovascular system, including changes in cardiac loading, energy substrate utilisation (via insulin resistance), tissue metabolism and systemic inflammation, all of which are believed to individually promote HF progression ${ }^{(42,43)}$. A recent large meta-analysis reports that the likelihood of displaying LV hypertrophy was higher in those with obesity ${ }^{(4)}$. Weight reduction is consistently associated with the decrease in LV mass ${ }^{(44,45)}$. Recent studies propose that improvements in neurohormonal and metabolic abnormalities associated with weight reduction such as sympathetic nervous system tone, renin-angiotensin-aldosterone system activation, insulin resistance and hyperleptinaemia may also play a role in reducing $\mathrm{LV}$ mass ${ }^{(46)}$. Indices of diastolic function have also consistently been reported to improve following weight loss ${ }^{(47)}$. Therefore, it could be the significant weight loss observed in the MRP group $(-13.5 \mathrm{~kg})$ that is driving the favourable cardiovascular changes.

The novelty of this secondary analysis from data from an RCT is that it is the first analysis to investigate the effects of a nutritionally balanced MRP on the plasma micronutrient concentrations of people with T2D and obesity and its subsequent impact on cardiovascular structure and function as determined by CMR. However, this secondary analysis uses a small sample size, and the null findings may be a result of a lack of statistical power (type II error) making it difficult to draw meaningful conclusions. We recommend further work to confirm the findings of the present analysis, which should include cohorts of people who have sub-optimal nutritional status.

\section{Conclusion}

The concentrations of vitamins $\mathrm{B}_{1}$ and $\mathrm{B}_{6}$ differ between people with $\mathrm{T} 2 \mathrm{D}$ and age- and sex-matched healthy controls. Among people with T2D, vitamin D concentration and LV mass:volume ratio are associated at baseline. A 12-week MRP induced a significant increase in several micronutrients (vitamins $\mathrm{B}_{1}, \mathrm{~B}_{6}, \mathrm{D}$ and $\left.\mathrm{C}\right)$. However, the improvement in circulating micronutrient levels showed no association with the observed improvements in cardiovascular structure or function following the 12-week MRP in people with T2D and obesity. This lack of association may be the consequence of the relatively good health status of this cohort and the small sample size. This area of research, therefore, warrants further investigation.

\section{Supplementary material}

The supplementary material for this article can be found at https://doi.org/10.1017/jns.2021.82.

\section{Acknowledgments}

The DIASTOLIC study was funded by the National Institute for Health Research, Research Trainees and Associates Coordinating Centre through a career development fellowship to G.P.M. (CDF 2014-07-045). G.S.C. was funded by the British Heart Foundation through a Clinical Research Training
Fellowship (FS/16/47/32190). This report is independent research supported by the National Institute for Health Research (NIHR Research Professorship, G.P.M. (RP-2017-08-ST2-007) which also funds E.M.B). Further support was provided by the NIHR Leicester Biomedical Research Centre, and the study utilised the NIHR Leicester Clinical Research Facility.

The views expressed in this publication are those of the author(s) and not necessarily those of the NHS, the National Institute for Health Research or the Department of Health and Social Care.

G. W. M. W. contributed to conception and design, contributed to analysis and interpretation, drafted the manuscript, made amendments to the manuscript after other author review, and accountable for all aspects of work ensuring integrity and accuracy. E. M. B. contributed to conception and design, contributed to analysis and interpretation, critically revised the manuscript, gave final approval and accountable for all aspects of work ensuring integrity and accuracy. G. P. M., G. S. G., T. Y. and M. J. D. contributed to conception and design, contributed to analysis and interpretation and critically revised the manuscript. E. R., J. H., S. A. and K. P. contributed to conception and design and critically revised the manuscript.

The authors declare that they have no conflicts of interest.

\section{References}

1. Diabetes UK (2019) Number of people with diabetes reaches 4.7 million. https://www.diabetes.org.uk/about_us/news/new-stats-peopleliving-with-diabetes (accessed April 2020).

2. Dunlay SM, Givertz MM, Aguilar D, et al. (2019) Type 2 diabetes mellitus and heart failure: a Scientific Statement from the American Heart Association and the Heart Failure Society of America: this statement does not represent an update of the 2017 ACC/AHA/ HFSA heart failure guideline update. Circulation 140, e294-e324.

3. Gulsin GS, Athithan L \& McCann GP (2019) Diabetic cardiomyopathy: prevalence, determinants and potential treatments. Ther $A d v$ Endocrinol Metab 10. doi: 10.1177/2042018819834869.

4. Athithan L, Gulsin GS, McCann GP, et al. (2019) Diabetic cardiomyopathy: pathophysiology, theories and evidence to date. World J Diabetes 10, 490.

5. Goldberg LR \& Jessup M (2006) Stage B heart failure: management of asymptomatic left ventricular systolic dysfunction. Circulation 113, $2851-2860$.

6. Physicians DiCWtACoC, Heart tISf, Transplantation L, et al. (2005) ACC/AHA 2005 guideline update for the diagnosis and management of chronic heart failure in the adult-summary article: a report of the American College of Cardiology/American Heart Association Task Force on Practice Guidelines (writing committee to update the 2001 Guidelines for the Evaluation and Management of Heart Failure). J Am Coll Cardiol 46, 1116-1143.

7. Sunkara A \& Raizner A (2019) Supplemental vitamins and minerals for cardiovascular disease prevention and treatment. Methodist Debakey Cardiovasc J 15, 179.

8. McKeag NA, McKinley MC, Harbinson MT, et al. (2017) Dietary micronutrient intake and micronutrient status in patients with chronic stable heart failure: an observational study. I Cardiovasc Nurs 32, 148-155.

9. Witte KK, Clark AL \& Cleland JG (2001) Chronic heart failure and micronutrients. J Am Coll Cardiol 37, 1765-1774.

10. Page G, Laight D \& Cummings M (2011) Thiamine deficiency in diabetes mellitus and the impact of thiamine replacement on glucose metabolism and vascular disease. Int J Clin Pract 65, 684-690.

11. Merigliano C, Mascolo E, Burla R, et al. (2018) The relationship between vitamin B6, diabetes and cancer. Front Genet 9, 388. 
12. Miyan Z \& Waris N (2020) Association of vitamin B12 deficiency in people with type 2 diabetes on metformin and without metformin: a multicenter study, Karachi, Pakistan. BMJ Open Diabetes Res Care 8, e001151.

13. Ozfirat Z \& Chowdhury TA (2010) Vitamin D deficiency and type 2 diabetes. Postgrad Med J 86, 18-25.

14. Simcox JA \& McClain DA (2013) Iron and diabetes risk. Cell Metab 17, 329-341.

15. Public Health England (2019) Diabetes prevalence and risk. Available at https://fingertips.phe.org.uk/profile/diabetes-ft/data (accessed February 2021).

16. Packer M (2018) Heart failure: the most important, preventable, and treatable cardiovascular complication of type 2 diabetes. Diabetes Care 41, 11-13.

17. Cascino TM, Hummel SL (2018) Nutrient deficiencies in heart failure: a micro problem with macro effects? Am Heart Assoc 7(17), e010447.

18. Via M (2012) The malnutrition of obesity: micronutrient deficiencies that promote diabetes. Int Sch Res Not 2012, Article ID 103472, 8 pages.

19. Dubey P, Thakur V \& Chattopadhyay M (2020) Role of minerals and trace elements in diabetes and insulin resistance. Nutrients 12, 1864.

20. Rosenblum H, Wessler JD, Gupta A, et al. (2020) Zinc deficiency and heart failure: a systematic review of the current literature. $J$ Card Fail 26, 180-189.

21. Vardi M, Levy NS \& Levy AP (2013) Vitamin E in the prevention of cardiovascular disease: the importance of proper patient selection. J Lipid Res 54, 2307-2314.

22. Gulsin GS, Brady EM, Swarbrick DJ, et al. (2019) Rationale, design and study protocol of the randomised controlled trial: Diabetes Interventional Assessment of Slimming or Training to Lessen Inconspicuous Cardiovascular Dysfunction (the DIASTOLIC study). BMJ Open 9, e023207.

23. Gulsin GS, Swarbrick DJ, Athithan L, et al. (2020) Effects of lowenergy diet or exercise on cardiovascular function in working-age adults with type 2 diabetes: a prospective, randomized, open-label, blinded end point trial. Diabetes Care 43, 1300-1310.

24. Public Health England (2016) Government recommendations for energy and nutrients for males and females aged 1-18 years and 19+ years. https://assets.publishing.service.gov.uk/government/uploads/ system/uploads/attachment_data/file/618167/government_dietary_ recommendations.pdf.

25. NHS UHL (2020) Blood sciences user handbook. In A User Guide for UHL Blood Science Pathology Services, vol. IN5501, pp. 137 [Leicester RW, NH Service Ed.].

26. Leicester UH (2019) Biochemistry and Haematology User Handbook. Leicester, UK: University Hospitals Leicester.

27. Valdés-Ramos R, Ana Laura G-L, Beatriz Elina M-C, et al. (2015) Vitamins and type 2 diabetes mellitus. Endocr Metab Immune Disord Drug Targets 15, 54-63.

28. Waheed P, Naveed AK \& Ahmed T (2013) Thiamine deficiency and its correlation with dyslipidaemia in diabetics with microalbuminuria. J Pak Med Assoc 63, 340-345.

29. DiNicolantonio JJ, Niazi AK, Lavie CJ, et al. (2013) Thiamine supplementation for the treatment of heart failure: a review of the literature. Congest Heart Fail 19, 214-222.
30. DiNicolantonio JJ, Liu J \& O'Keefe JH (2018) Thiamine and cardiovascular disease: a literature review. Prog Cardiovasc Dis 61, 27-32.

31. Rubí B (2012) Pyridoxal 5'-phosphate (PLP) deficiency might contribute to the onset of type I diabetes. Med Hypotheses 78, 179-182.

32. Mascolo E \& Vernì F (2020) Vitamin B6 and diabetes: relationship and molecular mechanisms. Int J Mol Sci 21, 3669.

33. Ahn HJ, Min KW \& Cho Y-O (2011) Assessment of vitamin B6 status in Korean patients with newly diagnosed type 2 diabetes. Nutr Res Pract 5, 34-39.

34. Velagaleti RS, Gona P, Chuang ML, et al. (2010) Relations of insulin resistance and glycemic abnormalities to cardiovascular magnetic resonance measures of cardiac structure and function: the Framingham Heart Study. Circ Cardiovasc Imaging 3, 257-263.

35. Pilz S, März W, Wellnitz B, et al. (2008) Association of vitamin D deficiency with heart failure and sudden cardiac death in a large cross-sectional study of patients referred for coronary angiography. J Clin Endocrinol Metab 93, 3927-3935.

36. Zittermann A, Schleithoff SS, Tenderich G, et al. (2003) Low vitamin D status: a contributing factor in the pathogenesis of congestive heart failure? J Am Coll Cardiol 41, 105-112.

37. Ameri P, Ronco D, Casu M, et al. (2010) High prevalence of vitamin D deficiency and its association with left ventricular dilation: an echocardiography study in elderly patients with chronic heart failure. Nutr Metab Cardiovasc Dis 20, 633-640.

38. Gulsin GS, Kanagala P, Chan DC, et al. (2019) Differential left ventricular and left atrial remodelling in heart failure with preserved ejection fraction patients with and without diabetes. Ther $A d v$ Endocrinol Metab 10. doi: 10.1177/2042018819861593.

39. Wilmot EG, Edwardson CL, Achana FA, et al. (2012) Sedentary time in adults and the association with diabetes, cardiovascular disease and death: Systematic review and meta-analysis. Diabetologia $\mathbf{5 5}$, 2895-2905.

40. Banerjee A, Chitnis U, Jadhav S, et al. (2009) Hypothesis testing, type I and type II errors. Ind Psychiatry J 18, 127.

41. Lauer MS, Anderson KM, Kannel WB, et al. (1991) The impact of obesity on left ventricular mass and geometry: the Framingham Heart Study. JAMA 266, 231-236.

42. Lavie CJ, Sharma A, Alpert MA, et al. (2016) Update on obesity and obesity paradox in heart failure. Prog Cardiovasc Dis 58, 393-400.

43. Paulus WJ \& Tschöpe C (2013) A novel paradigm for heart failure with preserved ejection fraction: comorbidities drive myocardial dysfunction and remodeling through coronary microvascular endothelial inflammation. J Am Coll Cardiol 62, 263-271.

44. Cuspidi C, Rescaldani M, Sala C, et al. (2014) Left-ventricular hypertrophy and obesity: a systematic review and meta-analysis of echocardiographic studies. J Hypertens 32, 16-25.

45. Rozenbaum Z, Topilsky Y, Khoury S, et al. (2019) Association of body mass index and diastolic function in metabolically healthy obese with preserved ejection fraction. Int J Cardiol 277, 147-152.

46. Alpert MA, Karthikeyan K, Abdullah O, et al. (2018) Obesity and cardiac remodeling in adults: mechanisms and clinical implications. Prog Cardiovasc Dis 61, 114-123.

47. Luaces M, Cachofeiro V, García-Muñoz-Najar A, et al. (2012) Anatomical and functional alterations of the heart in morbid obesity. Changes after bariatric surgery. Rev Esp Cardiol (Engl Ed) 65, 14-21. 